\title{
Editorial
}

\section{The neuropsychiatry in the age of subspecialization}

This issue of the European Journal of Psychiatry is devoted primarily to articles about patients with neuropsychiatric disorders. Psychiatry in the United States is increasingly being characterized by subspecialization. With the recent approval of Psychosomatic Medicine as a subspecialty in psychiatry, there are now five clinical subspecialties. Neuropsychiatry is another subspecialty which is seeking certification and has already received subspecialty status by a neurological review committee. Neuropsychiatry is generally defined as the psychiatric subspecialty that deals with the psychological and behavioral manifestations of brain disease and structural brain damage. In the latest issue of the Kaplan and Sadock's Comprehensive Textbook of Psychiatry, $8^{\text {th }}$ edition, neuropsychiatry is in the second section in the 2 volume set following the section on the neurosciences. As the most widely used textbook of psychiatry in the United States, this emphasizes the growing importance of both neuroscience and neuropsychiatry to the mainstream of psychiatric practice.

Since the early part of the $19^{\text {th }}$ century, when psychiatry differentiated itself as a medical specialty, the patients cared for by psychiatrists practicing in asylums had neuropsychiatric disorders. General paresis of the insane (neurosyphilis, as it was later identified), epilepsy, mental retardation, and mental disorders associated with alcohol abuse were all common conditions treated in $19^{\text {th }}$ century asylums. It wasn't until later in the $19^{\text {th }}$ and early $20^{\text {th }}$ century that Freud, Zung, Adler and other postulated psychological etiologies for disorders such as hysteria, phobias, depression, and anxiety disorders. I believe it is fair to say, however, that general psychiatry evolved from the recognition and care of patients with neuropsychiatric disorders.

Throughout the $20^{\text {th }}$ century, although there was a decrease in the emphasis of neuropsychiatric disorders in the mainstream of general psychiatry, there continued to be recognition by the early psychiatrists such as Adolph Meyer (1904), and Bleuler (1951), that many of the disorders treated by general psychiatrists, such as depression and anxiety, were often the consequence of brain disease. In his writings on the traumatic insanities, Meyer (1904) identified several disorders such as delirium, dementia, and aphasia which he felt were the direct result of brain injury. In keeping with his biopsychosocial view of most mental "reactions," however, he saw disorders such as manic depressive illness and paranoic conditions as arising from a combination of head injury, specifically citing left frontal lobe and cortical convexities, as well as family history of psychiatric disorders, prior life events, and prior history of psychiatric disorder. On the other hand, Emil Kraepelin (1921), stated that vascular disease such as atherosclerosis and the accompanying cerebral infarction may itself engender states of depression. Post (Post F. 1962) wrote that the association of brain infarction and first 
episodes of depressive disorder was so common that the causes for atherosclerotic vascular disease and depression might be etiologically linked.

Although there are a number of neuropsychiatric disorders, such as catastrophic reactions, pathological laughter and crying (recently termed involuntary emotional display disorder) and anosognosia, which are unique to patients with structural brain disorders, the topic of many recent neuropsychiatric studies has been the disorders treated by general psychiatrists. Thus, the article in this journal by Rao and colleagues examines depression following traumatic brain injury and its relationship to magnetic resonance spectroscopy signals in frontal cortex, basal ganglia and thalamus. Similarly, Levy and Fann report in this issue on depressions associated with the neurotoxic effects of drugs used in hematopoietic stem cell transplantation (HSCT) as well as the neurotoxic effect of associated radiation, infection, and immunosuppression therapy. Similarly, although both Spoletini et al. and Starkstein et al. have examined patients with Alzheimer's disease, the focus of their investigations is on the frequency, course and severity of delusions or apathy in these patients. Finally, Beglinger et al. report in this issue on the mood disorder and memory disorder provoked by damage to the mammillary bodies, the fornix and the mammilothalamic tracks. These structures comprise much of Papez circuit, which has long been hypothesized to be the neuroanatomical basis for normal emotional expression as well as a mediator for depression and amnestic disorders when the circuit is disrupted.

Neuropsychiatry, like other subspecialties in psychiatry, requires a specific body of knowledge and training which is outside the usual content of training programs in psychiatry. Neuropsychiatrists perform physical examinations and use a broad assessment of cerebral function including structural and functional neuroimaging and encephalographic studies, not just to rule out organic disease, but to see which specific disease is present and where it has affected the brain. Similarly, the neuropsychiatric brain is more complex than the general concept of brain function in psychiatry. The existence and disruption of neurotransmitters, neuroreceptors, and chemical imbalance form the basis for the most common psychiatric conceptualizations of the etiology of major psychiatric disorders. The neuropsychiatrist, on the other hand, is concerned with both the nature of the brain dysfunction and its location among brain circuits and systems. Thus, Beglinger et al. describes the importance of ablation of several structures central to the Papez circuit. Rao et al. describes the importance of Nacetyl aspartate (NAA) which is a marker of neuronal and axonal integrity and function (Bachelard H et al. 1993). Furthermore, Rao et al. found reduced NAA activity in the frontal lobe and basal ganglia suggesting abnormalities of the frontal-basal ganglia-thalamic circuit which has been postulated to mediate depression. Finally, Starkstein et al. hypothesized that the anterior cingulate -ventral globus pallidus- dorsal medial thalamus circuit may play a central role in the production of motivation and action.

In spite of the fact that neuropsychiatry utilizes different tools of investigation and different conceptualizations of the etiology of mental disorders, neuropsychiatry has focused, in large part, on mainstream psychiatric disorders. Thus, the point that I would like to emphasize in this special issue of the European Journal of Psychiatry is that the insights which may be gained from the study of patients with demonstrable brain pathology may shed light on the disorders confronting all psychiatrists. It is a hope of virtually all investigators conduct- 
ing neuropsychiatric research that these differences in the disorder being investigated and the different tools used in the study of neuropsychiatric patients will shed light on the underlying pathophysiology, pathoanatomy, and the neurocellular pathology of many of the mainstream psychiatric disorders.

The second point that I would like to make about the manuscripts in this issue is the diversity and therefore enormous amount of scientific research which is needed to reach the potential of neuropsychiatry to illuminate general mechanisms of psychopathology.

Beglinger et al. studied a patient with a craniopharyngioma, Levy and Fann studied patients with hematopoietic stem cell transplantation, Rao et al. studied patients with traumatic brain injury, and Starkstein et al. and Spoletini et al. examined patients with Alzheimer's disease. These disorders represent a small fraction of the large number of disorders studied by neuropsychiatrists including cerebrovascular disorders, brain tumors, multiple sclerosis, Parkinson's disease, Huntington's disease, and other neurodegenerative disorders, to name only a few. Thus, the investigation of these disorders and the identification of the mechanisms of depression, mania, panic disorder, cognitive dysfunction, as well as their treatments requires an enormous amount of investigation and time. Thus, we are at an early stage in the development and demonstration of the potential of neuropsychiatric disorders to illuminate etiology and specific targeted treatments of psychiatric disorders of interest to all psychiatrists. At the beginning of this editorial, I remarked about the growing interest in the findings coming from neuropsychiatric research. This has led to the success of specialty journals devoted to neuropsychiatry such as the Journal of Neuropsychiatry and Clinical Neuroscience as well as the textbooks of neuropsychiatry such as the American Psychiatric Publishing Textbook of Neuropsychiatry and Clinical Neurosciences (Yudofsky and Hales, eds), which is currently in its $5^{\text {th }}$ edition, and Neuropsychiatry (Schiffer, Rao and Fogel, eds) in its $2^{\text {nd }}$ edition. The most prominent psychiatric journals such as the British Journal of Psychiatry, American Journal of Psychiatry and the Archives of General Psychiatry contain articles about neuropsychiatric disorders in virtually every issue. Finally, analysis of the number of the publication in neuropsychiatry using Pub Med.gov as the online reference demonstrates an enormous increase in the publication rate for neuropsychiatric disorders such as Alzheimer's disease, stroke and traumatic brain injury which have increased from a total number of publications from 1980-1990 of 777 to1799 publications from 1991-2000.

Thus, there is tremendous potential for all psychiatrists to learn about the advances in neuropsychiatric disorders and the light they may shed on treatment and etiology of psychiatric disorders. It is my hope that this issue of the European Journal of Psychiatry will continue the integration of the subspecialty of neuropsychiatry into the scope of interest and knowledge of general practice psychiatrists. Perhaps the best demonstration of the utility of the integration of general psychiatry with neuropsychiatry is the progress that has been made in vascular depression. Fujikawa et al. (1993) demonstrated that in patients with the onset of depression after age 65, 93.7\% had evidence of vascular ischemia (silent cerebral infarction) on MRI scan. Likewise, the work of and Steffens and Krishnan (2002), Figiel (1990) and others (Coffey CE 1990, Tupler LA, et al. 2002) has demonstrated that hyperintensities reflecting primary vascular disease have been strongly associated with new onset depression in the elderly. For many years, these patients were treated by general practice psychiatrists. 
These depressions were generally thought to be the result of environmental circumstances such as loss of a spouse, change in life goals or involutional psychological mechanisms. We now recognize an important subclass of depressive disorders in the elderly, presumably provoked by cerebral ischemia, which constitutes an important interface between general psychiatry and neuropsychiatry.

I want to thank Professor Lobo for inviting this special issue as well as the authors who have spent the time and effort to bring to the readers of the European Journal of Psychiatry a glimpse at the breadth of interests and the tools being used in the investigation of neuropsychiatric disorders. These disorders led to the development of psychiatry as a specialty in medicine and, I believe, have now returned to a place in the mainstream of psychiatric investigation.

\section{References}

Bachelard H, Badar-Goffer R. NMR spectroscopy in neurochemistry. Review. J Neurochem 1993; 61(2): 412-429.

Bleuler EP. Textbook of Psychiatry. New York: Macmillan; 1951.

Coffey CE. Subcortical hyperintensity on magenetic resonance imaging: A comparison of normal and depressed elderly subjects. Am J Psychiatry 1990; 147: 187-189.

Figiel G, Krishnan RR, Doraiswamy PM, Rao VP, et al. Subcortical hyperintensities on brain magnetic resonance imaging: a comparison between late age onset and early onset elderly depressed subjects. Neurobiol Aging 1990; 26: 245-247.

Fujikawa T, Yamawaki S, Touhouda Y. Incidence of silent cerebral infarction in patients with major depression. Stroke 1993; 24: 1631-1634.

Kraepelin E. Manic Depressive Insanity and Paranoia. Edinburgh: E \& S Livingstone; 1921.

Meyer A. The anatomical facts and clinical varieties of traumatic insanity. Am J Insanity 1904; 60: 373-442.

Post F. The Significance of Affective Symptoms in Old Age. London: Oxford University Press; 1962.

Steffens DC, Krishnan KR, Crump C. Cerebrovascular disease and evolution of depressive symptoms in the cardiovascular health study. Stroke 2002; 33(6): 1636-1644.

Tupler LA, Krishnan KR, McDonald WM, Dombeck CB, et al. Anatomic location and laterality of MRI signal hyperintensities in late-life depression. J Psychosom Res 2002; 53(2): 665-676.

Robert G. Robinson, M.D.

The Paul W. Penningroth Professor an Head, Department of Psychiatry, The University of Iowa Carver College of Medicine, 200 Hawkins Dr, Iowa City IA 52242 ${ }^{1}$

1. This work was supported in part by the following NIH grants: MH52879, MH53592 and MH63405 